\title{
feature
}

\section{Vaccine outlooks}

\author{
After negative publicity and a series of setbacks over HIV/AIDS and influenza, the prospects for research \\ on new vaccines are improving
}

\section{Philip Hunter}

V accine research is at a crossroads between renewed optimism created by fundamental scientific advances, and pessimism from a series of scientific and publicity setbacks over the past decade. Early successes in the field against acute viral diseases, such as smallpox and polio, raised hopes that more serious infectious diseases could be controlled or even eradicated by vaccination, just as it was once thought that penicillin would eradicate major bacterial diseases such as tuberculosis and leprosy.

\section{...it became clear that many viruses were much tougher nuts to crack in terms of vaccine development...}

However, it became clear that many viruses were much tougher nuts to crack in terms of vaccine development than had been thought, and it also emerged that not all vaccines were equally safe. Indeed, mounting concerns over the safety of vaccines culminated in the infamous Wakefield paper published in the Lancet in 1998 that associated the MMR vaccine-against measles, mumps and rubella-with autism and inflammatory bowel disease in children. After several studies failed to reproduce these results, the Lancet eventually retracted this paper in 2010, but not before considerable damage had been done to public confidence in vaccination as a whole. Other factors have also sapped confidence in the field-notably the continuing failure to develop an effective vaccine against HIV/AIDS, 15 years after the first hopeful reports that a breakthrough might be imminent (Gorse et al, 1995).

Researchers have since developed a string of HIV/AIDS vaccine candidates, some of which have entered clinical trials, but none of which have been sufficiently efficient and safe. The gloom has deepened further after the failure of a vaccine candidate developed by the pharmaceutical company Merck in 2007, that had high hopes for success. This vaccine, called V520, used a weakened adenovirus that carries three HIV genes, to stimulate host production of T cells that it was hoped would kill HIV-infected cells. Early small trials had detected cellular immune responses, but these largely failed to materialize in a subsequent phase II clinical trial. The recombinant vaccine triggered a rapid immune response against itself that actually impaired the T-cell response against the HIV antigens. As a result, the trial was halted in September 2007 (Anon, 2007).

Controversially, it has since been suggested that V520 rendered some individuals more liable to subsequent infection, although views on this finding are polarized. According to Steven Patterson, a research fellow specializing in HIV at Imperial College, London, "there was a greater incidence of infection in those individuals who had immunity to adenovirus type 5 before vaccination. Some scientists argue that the numbers [of people who suffered infection during the trial] are relatively low, the results represent a statistical anomaly and that the effect gradually disappeared, suggesting that it was not a real effect. Others, including ourselves, think that in adenovirus type 5-immune individuals the vector activated pre-existing memory CD4 cells migrate to mucosal tissue. Then, because the virus preferentially replicates in activated CD4 T cells and the number of HIV susceptible cells is increased at the site of HIV infection, there is an increase in the number of infections. With time the activated cells return to a resting state which would explain why the effect of adenovirus vaccination gradually disappeared."

W hatever the truth in this case, it highlighted the setbacks in HIV/AIDS research and increased negative sentiments both among the public and, more crucially, funding agencies. "The devastating impact of HIV and its lethality have placed research on vaccines and other preventative measures under an unfamiliar spotlight," said Colonel Jerome Kim, HIV vaccines product manager for the US Army from the Walter Reed Army Institute of Research. "This has tended to exaggerate both the incremental successes and failures of HIV-1 vaccine research."

\section{"The devastating impact of HIV and its lethality have placed research on vaccines and other preventative measures under an unfamiliar spotlight"}

This might have contributed to a decline in public funding that has been combined with a continuing lack of investment from the private sector (AVERT, 2010). More worryingly, there have been signs that governments are withdrawing funding from vaccine research (Médecins Sans Frontières, 2009). In fact, the US government-through the National Institutes of Health-and the Bill and Melinda Gates Foundation-the charitable trust established in 1994 by Microsoft founder Bill Gates-accounted for $79 \%$ of the world's US $\$ 868$ million funding for HIV/AIDS vaccine research in 2008 (HIV Vaccines and Microbicides Resource Tracking Working Group, 2010). 


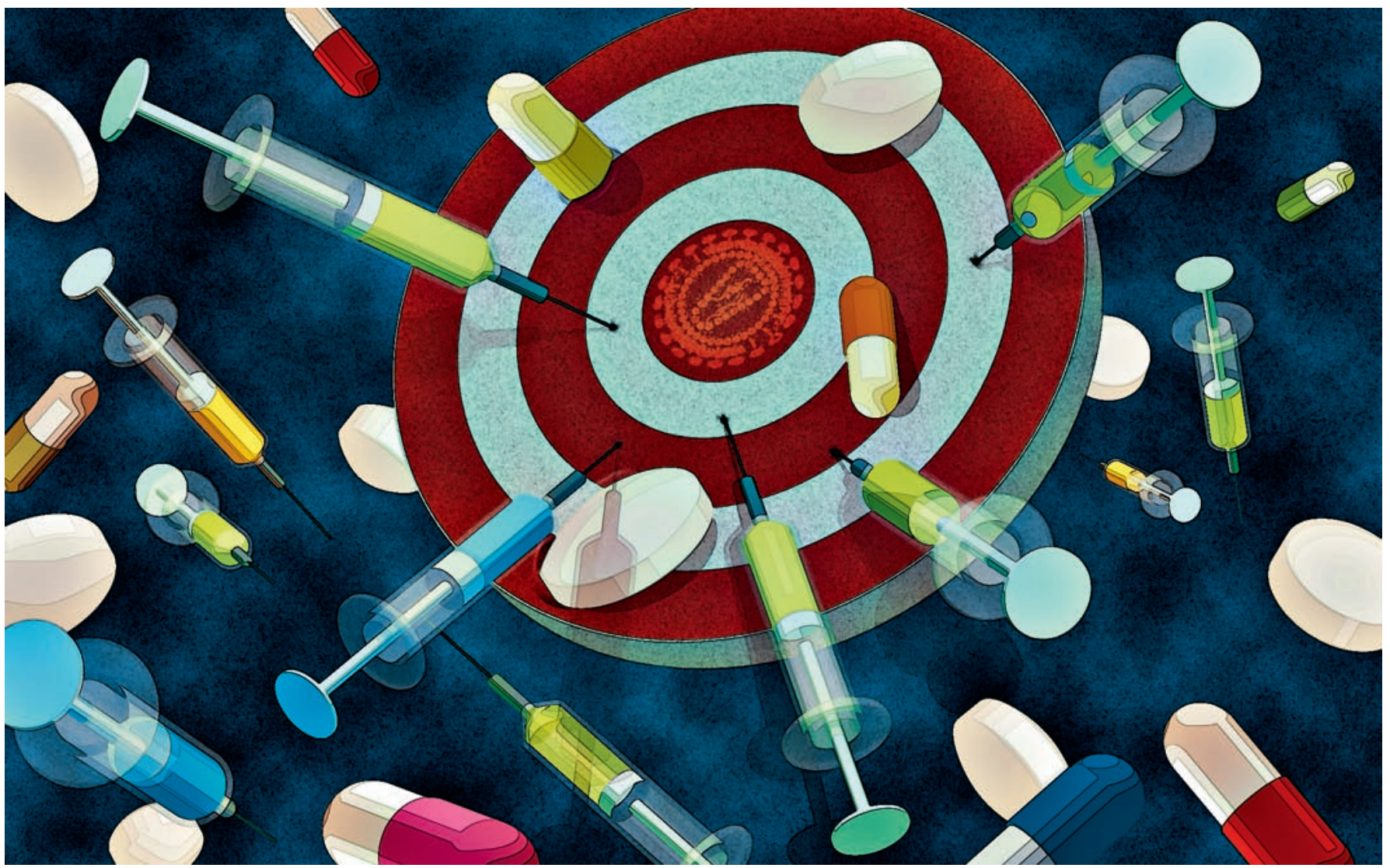

Other areas of vaccination research have also contributed to negative sentiments towards the field. A universal vaccine against influenza has proved similarly elusive, given the mutability of the virus. The recent swine flu pandemic - the severity of which fell short of many pessimistic expectationsleft many governments having spent huge sums on vaccines that they never needed. Furthermore, had swine flu become as virulent as it might have, these vaccine stockpiles might still have been only partially effective.

\section{More worryingly, there have been signs that governments are withdrawing funding from vaccine research}

All of these factors are fuelling groups who are opposed to vaccine research for various reasons, according to Joachim Hombach, at the World Health Organization's Initiative for Vaccine Research in Geneva. "There are certain groups, particularly in the industrialised world, that have an anti-vaccine attitude, and these kinds of cases find very fertile ground there," he said, referring in particular to the Lancet MMR article. "There is also a different story relating to general attitudes towards acceptance of absolutely no risk associated with vaccines or other medical interventions." This risk-averse culture exposes vaccines to public scrutiny when side effects occur during trials or afterwards, and has even been spreading to developing countries. "This creates a challenging climate for vaccine research," said Hombach.

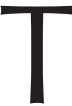

here are still strong grounds for optimism, as huge strides have been made in understanding the relationship between viruses and the immune response, which is more subtle and diverse than has been previously appreciated. In a sense, diseases such as polio represent the lowhanging fruit in the orchard of infectious disease; early successes in vaccine development have perhaps created a false sense of optimism. "Diseases that are more chronic, where you have a very delicate balance between the pathogen and the immune response, are very difficult to prevent with vaccines," Hombach said. "HIV is not the only one and there is also TB for instance, which is quite difficult. It is much easier to develop vaccines against acute diseases."

There is accordingly a need to educate the public about these difficulties, commented Tomáš Hanke, Nuffield professor of medicine specializing in HIV research at Oxford University. "Public confidence is a matter of public education and understanding of the process of scientific discovery," he said. "The more challenging the aim is, the more explaining the public needs."

Researchers disagree about the major problems hindering the development of new vaccines. In the case of HIV, Kim identified the elimination of $\mathrm{CD}_{4}{ }^{+}$helper $\mathrm{T}$ cells by the virus as one of the major problems, because these cells are at the centre of immune control and influence the activities of other cells. It is this disabling of helper T cells that weakens the immune response to other diseases in those who have AIDS.

Patterson believes that the greatest problem is the virus's mutability. "The ability of the virus to quickly mutate and escape from responses that are mounted against specific domains of the virus that are recognized by the immune system is, I think, the major hurdle that we need to overcome," he said. This 
is one reason why the traditional strategy of inducing protective antibodies by administering an attenuated virus has not worked for HIV.

$\mathrm{T}$ here are two further problems. First, important regions of the HIV virus are shielded by sugars and second, although antibodies that disable a broad range of HIV viruses have been identified, these tend to be produced too late in the immune response, when infection is already well established. For this reason, there has been increased focus on T-cell vaccines that can recognize and kill infected cells, rather than on efforts to prevent infection in the first place. Crucially though, as Patterson pointed out, this approach still faces the problem of mutation, because this can enable the virus to escape recognition by $T$ cells.

This, in turn, suggests that vaccines must target regions of the virus that are well conserved. "A normal T cell immune response tends to be against a small number of socalled dominant epitopes and in the case of HIV these are often against the more variable regions the virus can afford to mutate without any cost to itself," Patterson explained. "To avoid immune escape, we probably need to induce a $\mathrm{T}$ cell response against a number of conserved virus epitopes that the virus could not afford to mutate without severely impairing its replication capacity. I believe this aim is achievable."

\section{While the battle against HIV/ AIDS has been catching most of the headlines, a lesser known viral disease, dengue, has been rising quickly up the research agenda}

More fundamental research at the molecular level is needed to achieve this goal. Robin Weiss, professor of viral oncology at University College London, leads one team who are trying to identify antigens or targets that might one day become useful in vaccine design. Weiss does not claim to be near an imminent breakthrough, but he believes that a major step might be made soon towards developing a broad-spectrum vaccine that could prevent HIV infection in the first place. The problem, as Weiss pointed out, is not that individuals with HIV fail to produce antibodies, but that HIV elicits too many different ones, nearly all of which are ineffective. Only a few HIV-infected individuals produce potent antibodies and even then, these are often in insufficient concentrations. Now that the crystal structure of the potent antibodies has been defined, this vital molecular information could be used to design new vaccines that elicit production of these antibodies in the host.

Valuable information has also come from a US Army sponsored clinical trial in Thailand that studied more than 16,000 healthy individuals between 2003 and 2006. $A$ vaccine containing genetically engineered versions of three HIV genes was used, with an inert form of the bird virus canary pox as the vector. In December 2009, the sponsors reported that the rate of HIV infection among volunteers who received the experimental vaccine was $31 \%$ lower than among those who received a placebo (Rerks-Ngarm et al, 2009). "[The trial] showed, for the first time, that a vaccine is able to reduce the risk of HIV infection in humans," said Kim. "Although our results were modest, they are providing a great deal of information to inform the field. For example, the protection appeared highest at 6-12 months based on post-hoc analysis. If we can sustain or increase this effect, that would be a great accomplishment."

W hile the battle against HIV/ AIDS has been catching most of the headlines, a lesser known viral disease, dengue fever, has been rising quickly up the research agenda. Dengue fever is caused by four related strains of flavivirus that are transmitted by mosquitoes. The disease affects 50-100 million people annually, mostly in urban tropical areas, where an estimated 2.5 billion are at risk (Webster et al, 2009), largely because of growing urban populations. Attempts to develop a vaccine have been inhibited by the host immune response to the vaccine, according to Sarah Rowland-Jones, professor of immunology at the Weatherall Institute of Molecular Medicine in Oxford, UK. "It is a tough target for vaccine development principally because of the possibility that immune mechanisms contribute to pathogenesis, so researchers have to be particularly careful that a dengue vaccine does not make disease more likely because of the kind of immune response it stimulates, rather than leading to protection from infection," she said.

The fact that there are four viruses is another problem, especially as a vaccine protecting against one might actually prime individuals for another serotype. "The best hypothesis for severe dengue is that following a first infection, an individual gains immunity against that serotype but becomes more prone to a second infection with a different serotype," said Jeremy Farrar, Director of the Wellcome Unit in Vietnam and a professor of tropical medicine at Oxford University. "With that second infection against another serotype, more severe disease develops. Obviously this makes vaccine development difficult as one worries individuals will be 'primed' by the vaccine and, when they get a natural infection will have more severe disease."

\section{... the effect of each nucleotide change on virus activity is very small, but the cumulative impact of hundreds of such changes causes strong attenuation}

However, Farrar believes this problem has been fixed with the new generation of 'chimeric vaccines' that offer protection against all four serotypes. These are based on an exisiting vaccine against the related disease yellow fever, incorporating a part of the dengue virus that triggers an immune response. This chimeric approach has the potential to be extended to develop vaccines against other diseases.

This begs the question of why a dengue virus vaccine has not been developed already, given that the related yellow fever vaccine has been available for years. Part of the reason is that dengue is a tougher target, involving four serotypes, but it is also because its mortality is much lower than the $50 \%$ rate of yellow fever. "Dengue hasn't been top of the agenda because it hasn't caused so much mortality, but there is a lot of morbidity, and it puts a lot of stress on health authorities and has an epidemic potential," Hombach said. For this reason it has received more funding recently, with phase III clinical trials likely to begin soon, according to Farrar.

$\mathrm{n}$ entirely different approach for
engineering vaccines might also
be emerging. Traditional vaccines use mutated virus strains with limited replication abilities, in order to stimulate the immune system. The main drawback of this approach is that many attenuated strains fail to elicit adequate immunity, and it takes a long time to develop such strains. However, Eckard Wimmer and colleagues at Stony Brook University in New York, have developed a computer-aided approach to create attenuated strains without changing the 
composition and amino-acid sequence of the virus's proteins (Mueller et al, 2010).

They exploit the redundancy of the genetic code; 64 codons code for just 20 amino acids. As most amino acids are coded for by several codons, it is possible to introduce single-nucleotide changes without altering which proteins are expressed, thereby retaining all antigens that might generate an adaptive immune response. Crucially, however, this alters the expression of some genes, which reduces the ability of the virus to replicate. As Wimmer pointed out, the effect of each nucleotide change on virus activity is very small, but the cumulative impact of hundreds of such changes causes strong attenuation. "We call this 'death by a thousand cuts'," he said.

Moreover, subsequent natural mutations will probably change only one or two of these nucleotide substitutions back to the original, so the chance that the virus will regain its former virulence is very small. The greatest advantage of this approach, however, is speed. "Clearly, such recoded genomes can only be produced by chemical synthesis," said Wimmer. "They can be designed rapidly-much faster than any other live vaccine that was isolated after long trials of selection." This, argues Wimmer, makes the approach ideal for countering emerging pandemics when time is short, especially those caused by influenza. Indeed, Wimmer has demonstrated his approach by designing an influenza vaccine (Mueller et al, 2010).

Even if this synthetic approach can generate new vaccine candidates more quickly, it will still require development time and clinical trials to assess their efficacy and safety-as is the case for other emerging techniques. New knowledge and new technologies are filtering through to vaccine development; while the disappointments of the past have provided valuable lessons.

\section{REFERENCES}

Anon (2007) HIV vaccine failure prompts Merck to halt trial. Nature 44: 390

AVERT (2010) Funding for the HIV and AIDS Epidemic. Horsham, UK: Averting HIV and AIDS http://www.avert.org/aids-funding.htm

Gorse GJ, Rogers JH, Perry JE, Newman FK, Frey SE, Patel GB, Belshe RB (1995) HIV-1 recombinant gp160 vaccine induced antibodies in serum and saliva. The NIAID AIDS Vaccine Clinical Trials Network. Vaccine 13: 209-214

HIV Vaccines and Microbicides Resource Tracking Working Group (2010) Advancing the Science in a Time of Fiscal Constraint: Funding for HIV Prevention Technologies in 2009. New York, NY, USA: Global Advocacy for HIV Prevention. www.avac.org

Médecins Sans Frontières (2009) Punishing Success? Early Signs of a Retreat from Commitment to HIV/AIDS Care and Treatment. Geneva, Switzerland: Médecins Sans Frontières Mueller S, Coleman JR, Papamichail D, Ward CB, Nimnual A, Futcher B, Skiena S, Wimmer E (2010) Live attenuated influenza virus vaccines by computer-aided rational design.

Nat Biotechnol 28: 723-726

Rerks-Ngarm S et al (2009) Vaccination with ALVAC and AIDSVAX to prevent HIV-1 infection in Thailand. N Eng/ / Med 36: 2209-2220

Webster DP, Farrar J, Rowland-Jones S (2009) Progress towards a dengue vaccine. Lancet Infect Dis 9: 678-687

Philip Hunter is a freelance journalist in London, UK.

EMBO reports (2010) 11, 738-741. doi:10.1038/embor.2010.136

\section{Power to the scientists}

\section{New grant schemes are providing more support for young scientists at the start of their careers as independent researchers}

\section{Howard Wolinsky}

M aria Pia Cosma, whose research focuses on reprogramming stem cells, could have spent her entire career at the Telethon Institute of Genetics and Medicine in Naples, Italy - a nonprofit institute focused on researching human diseases. Instead, Cosma, 40, discovered a brave new world for early-career scientists when she was awarded a $€ 1.6$ million, five-year Starting
Independent Researcher Grant from the European Research Council (ERC; Brussels, Belgium). The offers came rolling in. She was stunned as Telethon offered her a substantial pay increase; the Universities of Dundee and Oxford, the Centre for Genomic Regulation (CRG) in Barcelona, and the National University of Ireland in Galway all offered her positions; Dundee even offered her a Chair.
Ultimately, Cosma opted to join the CRG as a senior group leader. Announcing the appointment in March this year, the CGR noted that Cosma is one of the few women in a senior position in Spain's biomedical sector, and also proudly pointed out that: "With Cosma on board, the CRG becomes one of Spain's life science research centres to receive the most ERC grants."

ERC grants are changing the game as they offer greater freedom to young investigators, but the ERC is not alone in boosting the prospects of young scientists. Other major research funding organizations are also now recognizing the need to support researchers early in their careers.

an Halliday, president of the European Science Foundation (ESF; Strasbourg,

France), explained that this trend is part of broader reforms being made to the academic system in Europe, especially in Germany, France and parts of Eastern Europe. These are challenging what he referred to as the traditional "Herr Professor" system in which "the older generation was perhaps too much in control of everything". Halliday said that the ERC has brought a breath of fresh air with its strong emphasis on young investigators. "There is an empowerment of the younger people against the structures that have been in place for perhaps rather too long," he said. "It isn't just 'Let's give money to the young people', but it's part of a general shake-up of the whole system in Europe. There's an ongoing effort to change quite substantially the structure of universities and how they're governed and the rules and regulations that govern them and so on."

He commented that the research establishment in Germany, for example, has been unsure how to handle major funding for ERC grantees. "It turned out that many German universities in fact had no method for taking a lot of money being given to young people," he said. "It had never happened before so there is no structure for that happening; that a 30-year-old could end up having several million dollars."

Halliday has also noted the contrast between the UK and other countries, from his involvement over the past four years in reorganizing physics departments across eight Scottish universities. "I was struck when I sat down at a meeting which consisted entirely of [continental] Europeans. My question was: 'What are you all doing in Scotland?' And their answer to a person was the fantastic contrast with the British system: at the age 